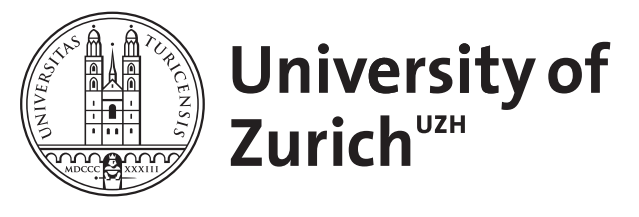

\title{
Surgical treatment of diabetic retinopathy
}

\author{
Helbig, Horst ; Sutter, Florian K P
}

\begin{abstract}
Several complications of advanced diabetic retinopathy can be treated surgically. Vitrectomy can clear media opacities, relieve traction on the retina, and makes adequate laser treatment of the retina possible. Removal of premacular vitreous may also improve diabetic macular edema. Instrumentation, understanding of the pathophysiology, surgical skills as well as supplementary pharmacotherapy have improved surgical results. Indications and timing for surgery have considerably changed in the past decades and the threshold for surgery has been continuously lowered, as vitrectomy became safer. The primary disease of the retinal microvasculature with capillary occlusion and retinal ischemia is still the limiting factor, responsible for disappointing functional results in anatomically successfully operated eyes
\end{abstract}

DOI: https://doi.org/10.1007/s00417-004-0977-9

Posted at the Zurich Open Repository and Archive, University of Zurich

ZORA URL: https://doi.org/10.5167/uzh-156040

Journal Article

Published Version

Originally published at:

Helbig, Horst; Sutter, Florian K P (2004). Surgical treatment of diabetic retinopathy. Graefe's archive for clinical and experimental ophthalmology = Albrecht von Graefes Archiv für klinische und experimentelle Ophthalmologie, 242(8):704-709.

DOI: https://doi.org/10.1007/s00417-004-0977-9 


\section{Horst Helbig Florian K. P. Sutter}

\section{Surgical treatment of diabetic retinopathy}

threshold for surgery has been continuously lowered, as vitrectomy became safer. The primary disease of the retinal microvasculature with capillary occlusion and retinal ischemia is still the limiting factor, responsible for disappointing functional results in anatomically successfully operated eyes.

H. Helbig ( ) F F. K. P. Sutter

Augenklinik,

UniversitätsSpital Zürich,

Frauenklinikstrasse 24, 8091 Zürich,

Switzerland

e-mail: horst.helbig@usz.ch

Tel.: +41-1-2554993

Fax: +41-1-2554472

\begin{abstract}
Several complications of laser treatment of the retina possible. Removal of premacular vitreous may also improve diabetic macular edema. Instrumentation, understanding of the pathophysiology, surgical skills as well as supplementary pharmacotherapy have improved surgical results. Indications and timing for surgery have considerably changed in the past decades and the
\end{abstract}

\section{Introduction}

Early documented attempts at vitreous surgery date back to Albrecht von Graefe in 1863 [11]. He predicted a vast field for surgical expertise in the future. It took a century to come up to his expectations, when in 1970 the first successful pars plana vitrectomy was performed by Machemer in an eye with diabetic vitreous hemorrhage [21]. Complications of proliferative diabetic retinopathy were then and still are important indications for vitreous surgery $[9,28]$.

In the meantime the introduction of laser therapy [18, 23 ] and the implementation of good metabolic control evolved as effective strategies to prevent the development of severe stages of diabetic retinopathy $[5,30]$. Nevertheless, a considerable number of eyes still progress and may become candidates for surgery. Progression of retinopathy may occur despite optimal treatment or because adequate therapy was not available.

Vitreous surgery addresses several problems of advanced diabetic retinopathy. Media opacities can be removed and tractively elevated retina reattached. More- over, removal of the vitreous has a stabilizing effect on the subsequent development of the proliferative process. In a vitrectomized eye there is no anatomical scaffold which could be used as a matrix for ingrowth of fibrovascular tissue into the eye. Relief of traction on retinal vessels may also improve blood flow within these vessels and reduce leakage. In addition, unrestricted circulation of fluid in the vitreous cavity after vitrectomy seems to improve the oxygen supply to the inner retina and prevents accumulation of vasoactive cytokines in the retina. In eyes with vitreous hemorrhage or tractional elevation of the retina it is often not possible to apply the necessary laser treatment to the retina. Intraoperative endolaser coagulation may therefore stabilize the proliferative process $[9,28]$. This review focuses on indications for vitreous surgery in proliferative diabetic retinopathy and on intraoperative strategies to avoid complications specific to vitreous surgery in diabetic retinopathy. 


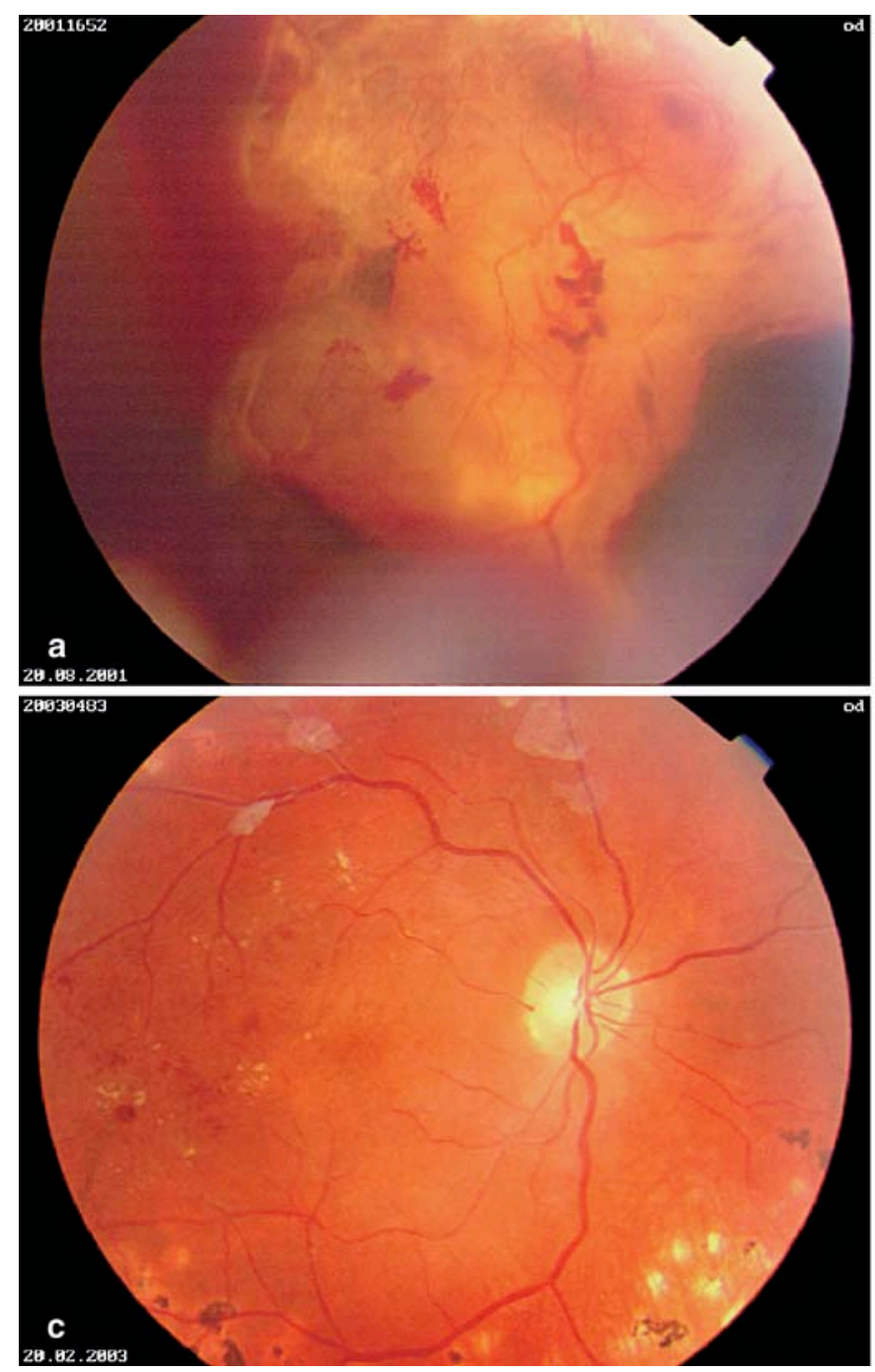

Fig. 1 a Dense subhyaloidal hemorrhage in an eye with extensive fibrovascular membranes. In the area without subhyaloidal blood the vitreous and the fibrovascular membranes are firmly attached to the retina, preventing penetration of blood between retina and vit-

\section{Indications for surgery}

\section{Media opacities}

Posterior segment neovascularizations mainly occur in eyes with an attached vitreous. Fibrovascular proliferations grow along the vitreoretinal interface and induce contraction and partial detachment of the vitreous. This contraction may tear the new vessels, leading to vitreous and subhyaloidal hemorrhage. Initially ophthalmologists recommended waiting 6-12 months before considering surgery for diabetic vitreous hemorrhage. The Diabetic Retinopathy Vitrectomy Study (DRVS) showed a clear benefit of early surgery for type 1 diabetics, who tend to develop more aggressive fibrovascular proliferations [6].
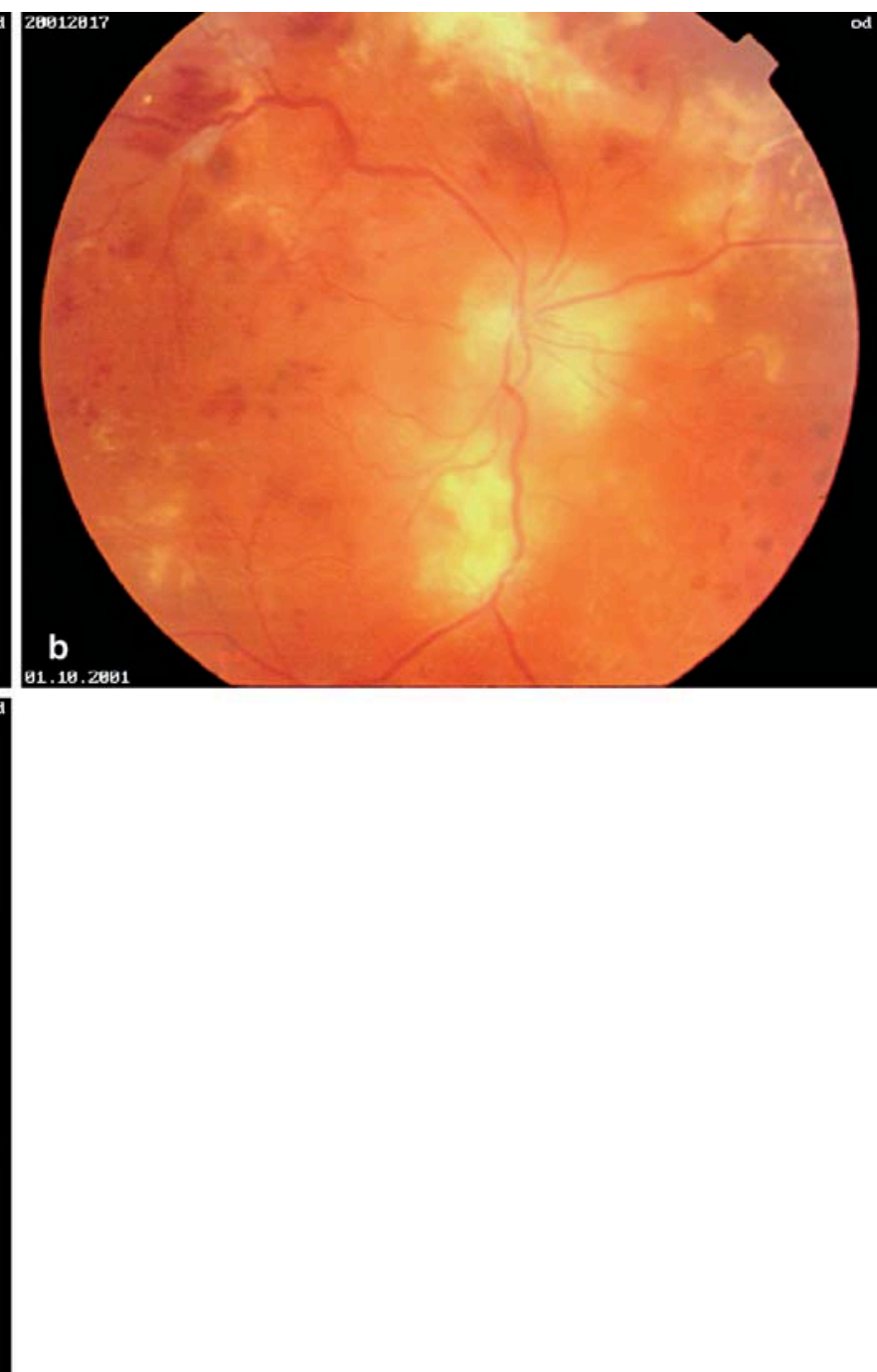

reous. $\mathbf{b}$ After vitreous surgery with removal of the membranes and endolaser, the retina is flat and extensive subretinal exudates due to tractive retinal edema are visible. c One year later the exudates are completely reabsorbed and VA has increased to 0.4

These eyes often experience progressive tractive complications while the retina cannot be visualized due to the vitreous hemorrhage. B-scan ultrasonography is an excellent tool for the detection of tractive fibrovascular membranes beneath the hemorrhage. These eyes require early surgery, while eyes without retinal traction may be followed for spontaneous clearing of the hemorrhage. Extensive subhyaloidal hemorrhage (Fig. 1) indicates an intact posterior hyaloid that may serve as an scaffold for further fibrovascular proliferations. Early surgery is recommended in these cases.

In eyes with vitreous hemorrhage and anterior segment neovascularizations, waiting for clearance of the hemorrhage may cause irreversible damage. Therefore, vitrectomy with intraoperative laser therapy should be per- 


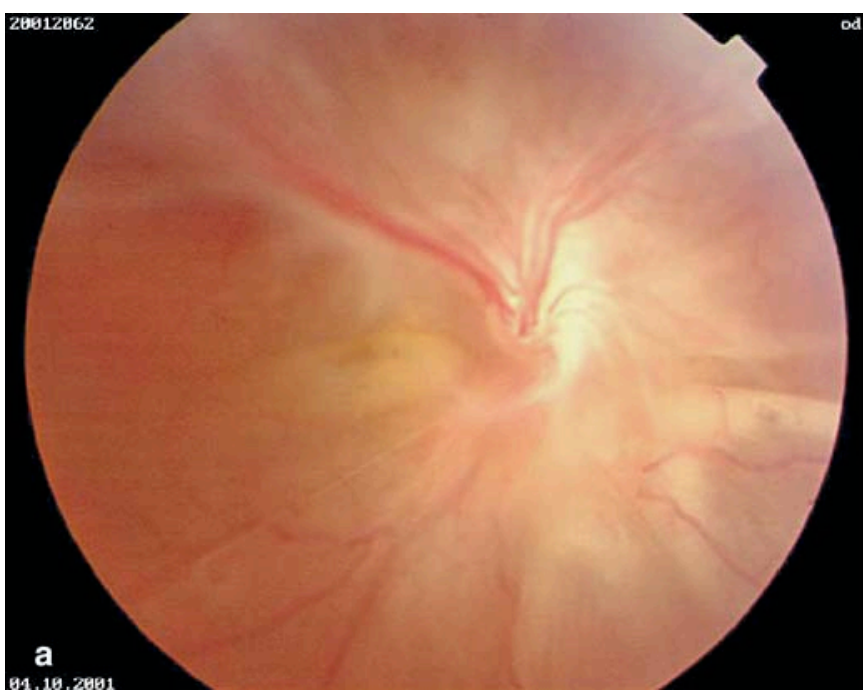

Fig. 2 a Tractional detachment of the macula. A fibrotic membrane has contracted, and pulled the retina over the optic disc and created a tractional retinal detachment of the macula. b One week after

formed early before irreversible obstruction of the chamber angle by fibrovascular tissue and neovascular glaucoma develops.

Other media opacities such as synchisis nivea may prevent adequate fundus visualization and a necessary laser treatment. Surgical removal of the vitreous opacities and endolaser treatment may be preferred to allow adequate control of the retinopathy [15].

Photocoagulation of the retina may not be possible in eyes with dense cataract, synechia, miosis, and active anterior or posterior segment neovascularizations. Performing only cataract surgery may even aggravate the neovascular process. In this situation combined cataract and vitreous surgery allowing adequate intraoperative laser therapy may be considered [2]. Alternatively, especially in cases with iris rubeosis, transscleral cryotreatment to the peripheral retina may be chosen to stabilize the anterior segment neovascular process before cataract surgery is performed [25]. Although some authors advocate routine retinal cryoablation several weeks before vitrectomy in diabetic eyes, this strategy is not generally recommended because of the intraocular inflammatory trauma induced by extensive cryotreatment [16].

\section{Vitreoretinal traction}

Tractional retinal detachment is not generally an urgent indication for surgery. Tractional retinal detachment with fibrotic, non-vascularized membranes outside of the macular region, especially nasally to the disc may be safely observed. Only $14 \%$ of eyes with extramacular tractional retinal detachment experienced a loss of vision

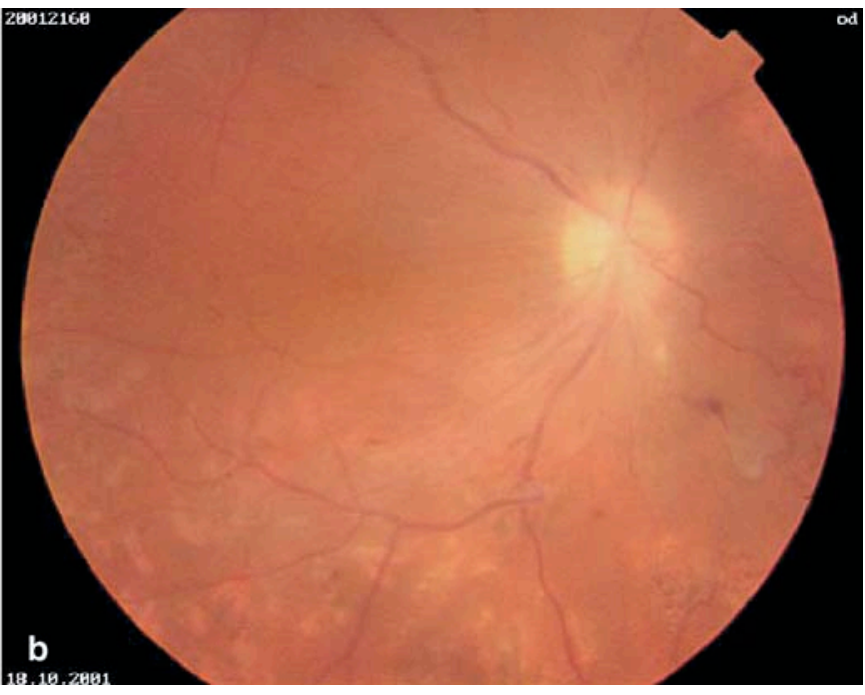

vitrectomy, removal of the preretinal membranes, and endolaser the retina is flattened. Visual acuity later improved to 0.6

within 1 year [4]. Risk factors for loss of vision were active neovascularization, concomitant vitreous hemorrhage and progression of the detachment. Tractional detachment involving or threatening the fovea is an indication for surgery. Eyes with recent circumscribed foveal detachment may have a good recovery of visual function (Fig. 2). Eyes with long-standing and widespread retinal detachment have a very poor functional prognosis, even if surgery is anatomically successful. Thus, it may be wise to avoid attempts at surgery with poor expectations and potential complications [12].

Laser treatment induces fibrotic regression of active neovascularizations. This fibrosis may exert increased tractive forces on the retina. Therefore, laser treatment may accelerate the development of tractional retinal detachment [8]. Eyes with extensive active fibrovascular proliferations should therefore be closely observed after photocoagulation. If laser treatment cannot be applied because of blood or fibrovascular tissue covering the retina, if regression of new vessels is not sufficient after laser treatment, or if traction is progressive, early vitreous surgery should be considered, before macular detachment develops.

Combined tractional and rhegmatogenous retinal detachment occurs if the contracting force of the fibrovascular complexes creates retinal tears (Fig. 3). This type of retinal detachment is rapidly progressive, the detached retina is more mobile and may attain a bullous and convex shape. Prompt surgery is usually necessary [29]. Technically this type of surgery may be demanding, because of difficulties separating the fibrovascular complexes from the mobile detached retina. 


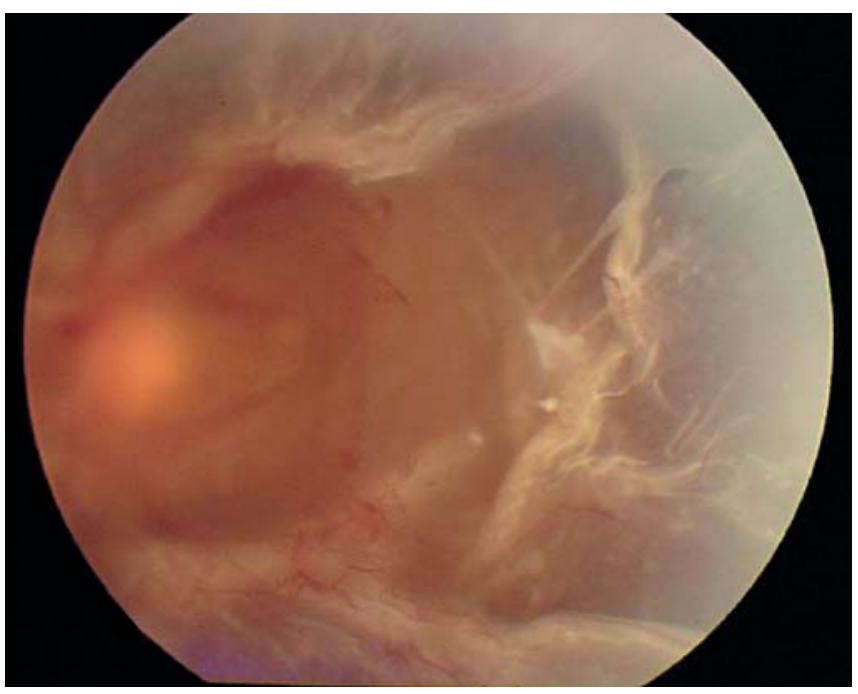

Fig. 3 a Tractive rhegmatogenous retinal detachment with a small tear created by tractional strands

\section{Diffuse macular edema}

Fibrovascular membranes exerting traction on the central retina may cause tractive macular edema. Surgical removal of these membranes is the treatment of choice for tractive diabetic macular edema (Fig. 1). Diabetic macular edema without visible traction is generally treated with photocoagulation. While focal diabetic macular edema responds well to focal laser treatment, the results of grid laser treatment for diffuse diabetic macula edema are unsatisfactory. Eyes with a detached posterior vitreous have a lower risk of developing diffuse macular edema [24]. Therefore vitreous detachment has been induced surgically as an attempt to treat diffuse diabetic macular edema. Indeed, a subgroup of eyes with a taut premacular posterior hyaloid seemed to benefit from vitrectomy [26]. This strategy has been further developed with the introduction of peeling of the internal limiting membrane for the treatment of diffuse diabetic macular edema [10]. It is however still controversial whether this approach really improves vision in these eyes. From the pathophysiological point of view, this procedure could relieve the retina from tractive forces, not visible biomicroscopically, caused by the preretinal vitreous and the internal limiting membrane. Optical coherence tomography (OCT) will possibly facilitate the visualization of the vitreoretinal interface and the identification of eyes with diffuse macula edema that should benefit from surgery. Another mechanism of action for vitrectomy in diffuse macular edema may be an improved supply of oxygen and nutrients from the vitreous cavity to the macula. Moreover, the taut condensed posterior premacular hyaloid may prevent the diffusion and cause the accumulation of cytokines produced by the ischemic retina which may lead to a diffuse breakdown of the inner blood-retinal barrier. After vitrectomy and ILM peeling these cytokines may escape easier into the vitreous cavity. Combinations of the mechanical surgical approach with pharmacological approaches (e.g., intravitreal triamcinolone) may further improve success rates for the treatment of diffuse diabetic macular edema [17].

\section{Avoiding complications of surgery}

The main goals of vitreoretinal surgery in diabetic retinopathy is to remove media opacities, completely relieve all tractional adhesions, and to apply adequate laser treatment to the retina. Two approaches can be used to remove the fibrovascular membranes. With the "delamination" or "en bloc" techniques the adhesions between the membrane and the retina are severed with horizontal cutting scissors and the membrane is removed in one piece. The "segmentation" technique involves cutting the membrane between two areas of adhesion with vertically cutting scissors. The remaining stumps are trimmed with the vitreous cutter [28]. Intravitreal gas tamponade and laser treatment can be used in cases where small retinal defects occur during preparation of the membranes.

Liquid silicone provides a permanent endotamponade of the vitreous cavity. It can be used in eyes with recurrent hemorrhage after vitrectomy to obtain a clear optic axis [14]. In eyes with multiple and large retinal defects, which can easily occur during preparation of tightly adhering membranes to a thin atrophic retina, liquid silicone may keep the retina attached. In this setting it is particularly important to remove all tractional tissue from the retina. Liquid silicone must not be used to push a tractively detached retina flat against the pigment epithelium and must not replace complete dissection of fibrovascular tissue. It is assumed that water-soluble growth factors reach high concentrations in the thin fluid film between retina and the silicone bubble. Therefore, under silicone there is probably an increased tendency for fibrovascular reproliferations [22].

Another indication for silicone is progressive anterior segment neovascularization, especially in aphacic and vitrectomized eyes [1]. In these eyes easy access for growth factors from the ischemic retina to the iris may lead to aggressive iris neovascularization. Instillation of liquid silicone prevents the easy diffusion from the retina to the anterior segment and in combination with intraoperative photocoagulation is an effective treatment option for progressive anterior segment neovascularization.

A serious complication of vitrectomy for diabetic retinopathy is the progression of iris rubeosis and the development of neovascular glaucoma [20]. Pathophysiologically two mechanisms are involved in the development of this complication. In eyes with active preretinal neovascularization these new vessels contribute to the 
supply of oxygen and nutrients to the inner retina. Surgical removal of these new vessels will aggravate retinal ischemia and stimulate the production of growth factors. Moreover, removal of the vitreous provides an easier diffusion of growth factors produced by the ischemic retina to the anterior segment of the eye and will increase the risk for anterior segment neovascularization. It is therefore important to extensively treat the ischemic retina with endolaser, especially in eyes where active preretinal neovascularizations are surgically removed [13]. If the retina is edematous, laser effects may be difficult to apply. Here it is helpful to inject perfluorcarbon liquids into the eye. Under perfluorocarbons the application of effective laser treatment to an edematous retina is much easier.

The management of the lens during and after vitreous surgery in diabetic eyes has changed considerably during the last decades. Complete removal of the anterior vitreous is not easily possible in phacic eyes and cataract develops rapidly after vitrectomy. The lens was therefore commonly removed intracapsularly during vitreous surgery. This procedure, however, led to a high rate of postoperative complications, especially the development of neovascular glaucoma [3]. The mechanism was probably the easy access of growth factors from the ischemic retina to the iris in an aphacic vitrectomized eye. If possible, intracapsular removal of the lens should therefore be avoided in diabetic eyes. Some surgeons successfully perform standard small incision extracapsular phacoemulsification with intraocular lens implantation simultaneously with vitreous surgery. The advantage of this combined procedures is to avoid a second surgical procedure and to allow more complete vitrectomy [19]. Posterior capsular opacification may occur earlier in vitrectomized diabetic eyes; primary posterior capsulotomy can therefore be considered if a combined vitreous and cataract surgery is performed. Disadvantages of the combined procedure may be an increased postoperative inflammatory response in the anterior segment with fibrinous exudation, precipitates on the IOL and formation of synechiae compared to a two-step procedure [27].

\section{Limitations of surgery}

With current advances in instrumentation, surgical skills, improved understanding of the pathophysiology, and supplementary medical drug treatment, vitreous surgery is approaching its limits in diabetic retinopathy. Surgery can safely clear media opacities and release traction on the retina in most cases. Postoperative complications such as severe intraocular inflammation and worsening of diffuse macular edema may be prevented by supplementary intravitreal pharmacotherapy. Intravitreal steroids have antiinflammatory and antiproliferative activity as well as the potential to dry out macular edema. Reliable efficacy and safety data on its use, however, are still to be provided. Fibrovascular reproliferation is a severe problem but rarely occurs if some basic rules are followed. The most significant problem in diabetic retinopathy is the vasoocclusive microvascular disease. Disappointing functional results after anatomically successful surgery are unfortunately common and are mostly due to retinal ischemia. No surgical approach so far enables effective reperfusion of occluded retinal capillaries. Our goals in the treatment of diabetic retinopathy must focus on strategies that prevent severe complications $[5,7,30]$ and therefore on making ourselves as vitreous surgeons dispensable.

\section{References}

1. Bartz-Schmidt KU, Thumann G, Psichias A, Krieglstein GK, Heimann K (1999) Pars plana vitrectomy, endolaser coagulation of the retina and the ciliary body combined with silicone oil endotamponade in the treatment of uncontrolled neovascular glaucoma. Graefes Arch Clin Exp Ophthalmol 237:969975

2. Benson GT, Flynn HW, Blankenship GW (1989) Posterior chamber intraocular lens implantation during diabetic pars plana vitrectomy. Ophthalmology 96:603-610

3. Blankenship GW (1980) The lens influence on diabetic vitrectomy results. Report of a prospective randomized study. Arch Ophthalmol 98:2196-2198
4. Charles S, Flinn CE (1981) The natural history of diabetic extramacular traction retinal detachment. Arch Ophthalmol 99:66-68

5. Diabetes Control and Complications Trial Research Group (1995) Progression of retinopathy with intensive versus conventional treatment in the Diabetes Control and Complications Trial. Diabetes Control and Complications Trial Research Group. Ophthalmology 102:647-661

6. Diabetic Retinopathy Vitrectomy Study Group (1990) Early vitrectomy for severe vitreous hemorrhage in diabetic retinopathy. Four-year results of a randomized trial: Diabetic Retinopathy Vitrectomy Study Report 5. Arch Ophthalmol 108:958-964
7. Donnelly R, Idris I, Forrester JV (2004) Protein kinase $\mathrm{C}$ inhibition and diabetic retinopathy: a shot in the dark at translational research. Br J Ophthalmol 88:145-151

8. Elliott A, Flanagan D (1990) Macular detachment following laser treatment of proliferative diabetic retinopathy. Graefes Arch Clin Exp Ophthalmol 228:438-441

9. Gandorfer A, Kampik A (2000) Pars plana vitrectomy in diabetic retinopathy. From pathogenetic principle to surgical strategy. Ophthalmologe 97:325-330 
10. Gandorfer A, Messmer EM, Ulbig MW, Kampik A (2000) Resolution of diabetic macular edema after surgical removal of the posterior hyaloid and the inner limiting membrane. Retina 20:126-133

11. von Graefe A (1863) Perforation von abgelösten Netzhäuten und Glaskörpermembranen. Graefes Arch Ophthalmol 9:84-104

12. Helbig H, Kellner U, Bornfeld N, Foerster MH (1996) Grenzen und Möglichkeiten der Glaskörperchirurgie bei diabetischer Retinopathie. Ophthalmologe 93:647-654

13. Helbig H, Kellner U, Bornfeld N, Foerster MH (1998) Rubeosis iridis after vitrectomy for diabetic retinopathy. Graefes Arch Clin Exp Ophthalmol 236:730-733

14. Hoerauf H, Roider J, Bopp S, Lucke K, Laqua H (1995) Endotamponade with silicon oil in severe proliferative retinopathy with attached retina. Ophthalmologe 92:657-662

15. Ikeda T, Sawa H, Koizumi K, Yasuhara T, Kinoshita S, Tano Y (1998) Vitrectomy for proliferative diabetic retinopathy with asteroid hyalosis. Retina 18:410-414

16. Jaccoma EH, Conway BP, Campochiaro PA (1985) Cryotherapy causes extensive breakdown of the blood-retinal barrier-a comparison with argon photocoagulation. Arch Ophthalmol 103:1728-1730
17. Jonas JB, Hayler JK, Sofker A, PandaJonas S (2001) Intravitreal injection of crystalline cortisone as adjunctive treatment of proliferative diabetic retinopathy. Am J Ophthalmol 131:468471

18. Körner F, Körner U (1988) Diabetische Retinopathie und Photokoagulation. Enke, Stuttgart

19. Lahey JM, Francis RR, Kearney JJ (2003) Combining phacoemulsification with pars plana vitrectomy in patients with proliferative diabetic retinopathy: a series of 223 cases. Ophthalmology 110:1335-1339

20. Laqua H (1980) Rubeosis iridis following pars plana vitrectomy. Klin Monatsbl Augenheilkd 177:24-30

21. Machemer R, Buettner H, Norton RWD, Parel JM (1971) Vitrectomy: a pars plana approach. Trans Am Acad Ophthalmol Otolaryngol 75:813-820

22. Messmer E, Bornfeld N, Oehlschlager U, Heinrich T, Foerster MH, Wessing A (1992) Epiretinal membrane formation after pars plana vitrectomy in proliferative diabetic retinopathy. Klin Monatsbl Augenheilkd 200:267-272

23. Meyer-Schwickerath G (1959) Lichtkoagulation. Enke, Stuttgart

24. Nasrallah FP, Jalkh AE, Van Coppenolle F, Kado M, Trempe CL, McMeel JW, Schepens CL (1988) The role of the vitreous in diabetic macular edema. Ophthalmology 95:1335-1339
25. Pauleikhoff D, Engineer B, Wessing AS (1997) Die Kryokoagulation in der Therapie der proliferativen diabetischen Retinopathie. Klin Monatsbl Augenheilkd 210:147-152

26. Pendergast SD, Hassan TS, Williams GA, Cox MS, Margherio RR, Ferrone PJ, Garretson BR, Trese MT (2000) Vitrectomy for diffuse diabetic macular edema associated with a taut premacular posterior hyaloid. Am J Ophthalmol 130:178-186

27. Shinoda K, O'Hira A, Ishida S, Hoshide M, Ogawa LS, Ozawa Y, Nagasaki K, Inoue M, Katsura H (2001) Posterior synechia of the iris after combined pars plana vitrectomy, phacoemulsification, and intraocular lens implantation. Jpn J Ophthalmol 45:276-280

28. Smiddy WE, Flynn HW Jr (1999) Vitrectomy in the management of diabetic retinopathy. Surv Ophthalmol 43:491507

29. Thompson JT, de Bustros S, Michels RG, Rice TA (1987) Results and prognostic factors in vitrectomy for diabetic traction-rhegmatogenous retinal detachment. Arch Ophthalmol 105:503507

30. United Kingdom Prospective Diabetes Study Group (1998) Intensive bloodglucose control with sulphonylureas or insulin compared with conventional treatment and risk of complications in patients with type 2 diabetes (UKPDS 33). UK Prospective Diabetes Study (UKPDS) Group. Lancet 352:837-853 